\section{EZH2 couples pancreatic regeneration to neoplastic progression}

Jon Mallen-St. Clair 1,5,6 Rengin SoydanerAzeloglu, ${ }^{1,5}$ Kyoung Eun Lee, ${ }^{1,7}$ Laura Taylor, ${ }^{1}$ Alexandra Livanos, ${ }^{2}$ Yuliya Pylayeva-Gupta, ${ }^{1}$ George Miller, ${ }^{3}$ Raphaël Margueron,, 1,8 Danny Reinberg, ${ }^{1,4}$ and Dafna Bar-Sagi ${ }^{1,9}$

${ }^{1}$ Department of Biochemistry, New York University School of Medicine, New York, New York 10016, USA; ${ }^{2}$ New York University School of Medicine, New York, New York 10016, USA; ${ }^{3}$ Department of Surgery, New York University School of Medicine, New York, New York 10016, USA; ${ }^{4}$ Howard Hughes Medical Institute, New York, New York 10016, USA

Although the polycomb group protein Enhancer of Zeste Homolog 2 (EZH2) is well recognized for its role as a key regulator of cell differentiation, its involvement in tissue regeneration is largely unknown. Here we show that EZH2 is up-regulated following cerulein-induced pancreatic injury and is required for tissue repair by promoting the regenerative proliferation of progenitor cells. Loss of EZH2 results in impaired pancreatic regeneration and accelerates $\mathrm{KRas}^{\mathrm{G12D}}$-driven neoplasia. Our findings implicate EZH2 in constraining neoplastic progression through homeostatic mechanisms that control pancreatic regeneration and provide insights into the documented link between chronic pancreatic injury and an increased risk for pancreatic cancer.

Supplemental material is available for this article.

Received October 24, 2011; revised version accepted February 2, 2012.

Tissue injury instigates a regenerative program, the purpose of which is to restore structure and function of the damaged organ. The proper execution of this program is critical for the maintenance of tissue homeostasis. Impaired regeneration can lead to tissue atrophy, and conversely, sustained engagement of tissue repair mechanisms has been linked to neoplasia (Coussens and Werb 2002). Understanding tissue response to injury in the pancreas is particularly relevant, as chronic exposure to injury and regeneration in the pancreas is associated with high neoplastic risk (Whitcomb 2004). The exocrine component of the adult pancreas consists predominantly

[Keywords: EZH2; pancreas; regeneration; cancer]

${ }^{5}$ These authors contributed equally to this work.

Present addresses: ${ }^{6}$ Department of Surgery, Division of Head and Neck Surgery, University of California at Los Angeles, Los Angeles, CA 90095, USA; ${ }^{7}$ Abramson Family Cancer Research Institute, Howard Hughes Medical Institute, Perelman School of Medicine, University of Pennsylvania, Philadelphia, PA 19104, USA; ${ }^{8}$ Institut Curie, 75005 Paris, France. Corresponding author.

E-mail dafna.bar-sagi@nyumc.org.

Article is online at http://www.genesdev.org/cgi/doi/10.1101/gad.181800.111. of acinar cells that display a robust capacity to undergo regeneration and renewal in response to insults that disrupt tissue integrity. Several signaling pathways, including TGF $\alpha$, Hedgehog, and Notch, have been implicated in regulation of acinar cell regeneration (Koizumi et al. 2003; Thayer et al. 2003; Jensen et al. 2005; Siveke et al. 2008). However, much remains to be elucidated concerning cellular and molecular mechanisms underlying pancreatic regeneration.

In the past decade, there has been an increasing appreciation of the critical role of epigenetic modifications in controlling the activity of regulatory genes that are involved in lineage specification, differentiation, and tissue renewal. In the present study, we sought to determine the role of Enhancer of Zeste Homolog 2 (EZH2), a polycomb group protein (PcG) and a member of the polycomb repressor complex 2 (PRC2), in the regeneration process that ensues following pancreatic injury. EZH2 catalyzes the trimethylation of histone H3-K27 (H3K27me3), a post-translational modification that has been widely implicated in epigenetic suppression of gene expression. The enzymatic catalysis of this modification is mediated by a specific domain within the protein known as the SET domain (Margueron and Reinberg 2011). We show that EZH2 is transiently up-regulated during pancreatic regeneration. Increased expression of EZH2 controls the proliferative expansion of pancreatic progenitor cells through the repression of the $p 16^{\text {Ink } 4 a}$ locus. Loss of EZH2 is associated with a failure to accomplish pancreatic regeneration and marked acceleration of oncogenic KRas-driven neoplasia. Our findings implicate EZH2 in linking pancreatic regenerative proliferation to oncogenic transformation.

\section{Results and Discussion}

To examine the role of EZH2 in tissue regeneration, we exploited an established experimental model of pancreatic epithelial injury and repair involving repetitive administration of supraphysiological levels of cerulein, a decapeptide analog of the pancreatic secretagogue cholecystokinin (Materials and Methods; Jensen et al. 2005; Fendrich et al. 2008; Siveke et al. 2008). Consistent with published observations, cerulein treatment induced severe exocrine pancreatic injury, which was readily observed within 1-3 d after the final administration and was histologically characterized by disordered acinar structure and abundant presence of metaplastic lesions (Fig. 1A). Within the following 7-9 d, injury was gradually resolved, and the exocrine compartment reached a fully restored appearance (Fig. 1A).

We next examined the temporal and spatial regulation of EZH2 levels in cerulein-induced pancreatic injury and regeneration. Whereas EZH2 was barely detectable in the uninjured pancreas, there was a pronounced up-regulation of EZH2 in response to cerulein administration, which peaked at day 3 and returned to near baseline by day 9 (Fig. 1B,C). In parallel, levels of H3K27me3 increased transiently (Fig. 1C). Notably, no changes in other components of PRC2, such as EZH1 and SUZ12, were observed, suggesting a unique role for $\mathrm{EZH} 2$ in the regenerative process (Fig. 1C). Of note, in the present study, cerulein administration was carried out using a 3 -wk protocol to 
Mallen-St. Clair et al.

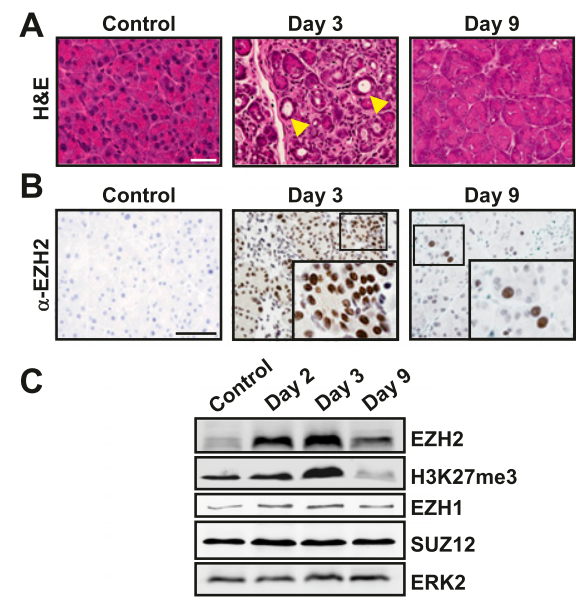

Figure 1. EZH2 is up-regulated during pancreatic regeneration. $(A)$ Histological progression of cerulein-induced injury and regeneration. H\&E-stained sections from tissues harvested at indicated intervals following final injection of cerulein. Metaplastic structures are shown by arrowheads. Bar, $50 \mu \mathrm{m}$. (B) Immunohistochemical analysis of EZH2 reveals a transient increase in nuclear EZH2 levels during the early stage of regeneration. (Insets) Higher magnification of the boxed areas in the corresponding panels. Bar, $50 \mu \mathrm{m}$. The images in $A$ and $B$ are representative of data obtained from five animals. $(C)$ Western blots demonstrating a concordance between EZH2 and H3K27me3 up-regulation upon injury. Levels of other components of PRC2 (EZH1 and SUZ12) do not change during exocrine regeneration. ERK2 serves as loading control. Data shown are representative of three independent experiments.

maximize the extent of injury (Materials and Methods). However, the up-regulation of EZH2 did not require prolonged exposure to cerulein. In fact, EZH2 induction was observed even after $2 \mathrm{~d}$ of cerulein injection (data not shown), underscoring the tight link between pancreatic injury and EZH2 accumulation. An increase in EZH2 upregulation was also detected in human pancreatitis samples, underscoring the pathophysiological relevance of EZH2 modulation during pancreatic injury (Supplemental Fig. 1).

The overt increase in EZH2 that accompanies the regenerative response to pancreatic injury suggests that EZH2 may play a role in this process. To investigate this possibility, we crossed mice harboring a conditional knockout allele of the SET domain of EZH2 (EZH2 $2^{f l / f 1}$ ) with p48-Cre mice, which results in EZH2 gene deletion in pancreatic epithelium starting at embryonic day 8.5 (Chen et al. 2009). Cre-mediated excision of the SET domain in the compound mice (hereafter referred to as p48-Cre;EZH2 ${ }^{\Delta S E T}$ ) was verified by allele-specific PCR genotyping of pancreatic tissue (Supplemental Fig. 2A). Western blot analysis of pancreatic lysates from $p 48$ Cre;EZH2 $2^{\triangle S E T}$ confirmed the lack of EZH2 expression (Supplemental Fig. 2B), consistent with reports that the deletion of the SET domain compromises the accumulation of EZH2 (Su et al. 1999). p48-Cre;EZH2 ${ }^{\Delta S E T}$ mice were born at the expected frequency and showed no evidence of gross changes in pancreatic development (Supplemental Fig. 2C) or cytoarchitecture (Supplemental Fig. 2D); displayed no signs of pancreatic insufficiency, as determined by normal fasting glucose levels (Supplemental Fig. 2E); and gained weight normally (data not shown). These data indicate that EZH2 is not essential for pancreatic development and physiology.
To interrogate the role of EZH2 in pancreatic regeneration, we monitored the regenerative response of $p 48$ $C r e ; E Z H 2^{\triangle S E T}$ mice to cerulein-induced injury. The predicted failure to up-regulate EZH2 in these animals was ascertained by immunohistochemistry and Western blotting (Supplemental Fig. 3A). The loss of EZH2 was not compensated for by an increase in its homolog, EZH1 (Supplemental Fig. 3B). p48-Cre;EZH $2^{\Delta S E T}$ mice displayed a defective regenerative response to cerulein-induced injury, as evident from the persistence of metaplastic lesions and failure to regain acinar architecture on day 9 after injury (Fig. 2). Indeed, pancreatic parenchyma of p48-Cre;EZH2 ${ }^{\triangle S E T}$ mice did not regain normal appearance even at $3 \mathrm{wk}$ after the final administration of cerulein (Supplemental Fig. 4). These observations suggest a role for $\mathrm{EZH} 2$ in regulating the regenerative capacity of the exocrine pancreas.

Lineage tracing and genetic and physiological studies (Jensen et al. 2005; Desai et al. 2007) have delineated a sequence of distinct cellular activities that are required for pancreatic regeneration following cerulein-mediated injury (Supplemental Fig. 5A). Thus, surviving acinar cells undergo dedifferentiation to generate metaplastic epithelium that expresses the early developmental marker PDX1 (Jensen et al. 2005). Typically, this developmental reprogramming takes place within 1-2 d post-injury and is accompanied by a transient cell cycle re-entry and proliferative expansion of metaplastic epithelium. Subsequently, acinar cell mass is restored through reactivation of an exocrine differentiation program. To determine the mechanism underlying the impaired exocrine regeneration caused by loss of EZH2, we investigated the contribution of $\mathrm{EZH} 2$ to the aforementioned steps of the regenerative process. Morphometric and biochemical analyses of amylase levels on day 1 following cerulein injury showed no difference in the extent of amylase loss between $E Z H 2^{f l / f 1}$ and p48-Cre;EZH2 $2^{\Delta S E T}$ mice, indicating that absence of EZH2 does not influence the severity of initial pancreatic injury (Fig. 3A; Supplemental Fig. 5B). The initial stromal response to pancreatic injury was also comparable between $E Z H 2^{f 1 / f 1}$ and p48-Cre;EZH2 $2^{\Delta S E T}$

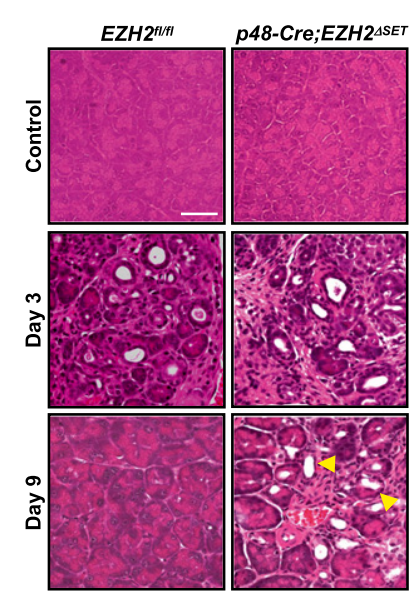

Figure 2. Impaired regeneration of the exocrine pancreas in $p 48$ Cre;EZH2 ${ }^{\Delta S E T}$ mice. H\&E-stained sections from tissues harvested at the indicated intervals following final injection of cerulein. Persistent metaplastic lesions in p48-Cre;EZH2 ${ }^{\Delta S E T}$ mice on day 9 are indicated by arrowheads. Images shown are representative of data obtained from three mice per genotype. Bar, $50 \mu \mathrm{m}$. 
A

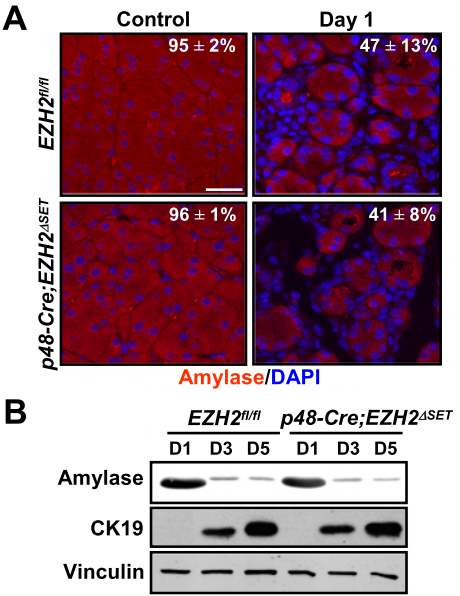

Figure 3. EZH2 deficiency does not affect cerulein-induced exocrine pancreatic injury or acinar cell dedifferentiation. $(A)$ Quantitative immunofluorescence analysis of amylase expression (see the Supplemental Material for details) demonstrating an equivalent level of acinar compartment depletion in $E Z H 2^{f 1 / f 1}$ and $p 48$ Cre;EZH2 $2^{\triangle S E T}$ mice. Acini are marked by amylase staining in red, and nuclei are marked by DAPI in blue. Images shown are representative of data obtained from two mice per genotype. Results represent the means \pm range. Bar, $50 \mu \mathrm{m}$. $(B)$ Cultured acinar cells from $E Z H 2^{f l / f 1}$ and $p 48$-Cre;EZH $2^{\Delta S E T}$ pancreata were harvested on days 1,3 , and 5 after isolation. Western blot analysis of cell lysates displays similar kinetics of dedifferentiation as assessed by downregulation of amylase expression and up-regulation of CK19. Vinculin serves as loading control. Results shown are representative of data obtained from three mice per genotype.

mice, as shown by trichrome $\mathrm{C}$ staining for fibrosis and CD45 staining for immune cell infiltration (Supplemental Fig. 5C,D).

Next, we sought to determine whether EZH2 is required for formation of metaplastic epithelial intermediates. A defining histological feature of this dedifferentiation process is acquisition of duct-like characteristics by acinar cells. Both EZH2 $2^{f l / f 1}$ and p48-Cre;EZH2 $2^{\Delta S E T}$ mice displayed abundant metaplastic lesions, suggesting that EZH2 is not essential for metaplastic conversion (Fig. 2; Supplemental Fig. 5E). Examining the generation of metaplastic epithelium using an established acinar cell culture model (Sawey et al. 2007) has provided further evidence in support of this conclusion. When placed in Matrigel, clusters of acinar cells lose their characteristic grape-like appearance within 3-5 d and acquire a spheroid morphology similar to that assumed by duct epithelial cells (Supplemental Fig. 6). This morphological transition is accompanied by a decrease in expression of amylase and a concomitant increase in expression of ductal cell marker CK19 (Sawey et al. 2007). Using the same criteria, Figure 3B illustrates that the rate and extent of acinar cell dedifferentiation in vitro were not affected by EZH2 deficiency.

Having established that EZH2 is dispensable for pancreatic injury and dedifferentiation following cerulein administration, we proceeded to investigate whether EZH2 may play a role in the proliferative expansion of the metaplastic epithelium. The kinetics of cell cycle reentry after cerulein treatment was assessed by immunofluorescence staining for $\mathrm{Ki}-67$. A robust proliferative response was observed in $E Z H 2^{f l / f 1}$ mice at day 3 posttreatment (Fig. 4A; Supplemental Fig. 7A), in agreement with published reports of mitotic activity during ceru- lein-induced pancreatic regeneration (Jensen et al. 2005; Fendrich et al. 2008). In contrast, there was a pronounced decrease in proliferating cells in regenerating pancreata of p48-Cre;EZH2 $2^{\Delta S E T}$ mice (Fig. 4A; Supplemental Fig. 7A). Double immunofluorescence analysis revealed that the vast majority of the proliferating cells at this stage of regeneration were positive for the pancreatic progenitor marker PDX1 (Supplemental Fig. 7B), suggesting that $\mathrm{EZH} 2$ function is required for the proliferative expansion of the progenitor-like metaplastic epithelium. In line with this idea, EZH2 up-regulation upon cerulein-induced injury is observed in the PDX1-positive metaplastic epithelium (Supplemental Fig. 7C). Hence, impaired regeneration caused by EZH2 deficiency might reflect the compromised proliferation of metaplastic cells and, consequently, the inability to effectively repopulate the injured pancreas.

To understand how loss of EZH2 might constrain proliferation of metaplastic progenitor cells, we pursued the possible involvement of the CDK inhibitor p16 ${ }^{\mathrm{INK} 4 \mathrm{~A}}$. Recent studies have linked EZH2 expression to suppression of $16^{\mathrm{INK} 4 \mathrm{~A}}$ expression (Chen et al. 2009; Ezhkova et al. 2009). In regenerating pancreata of $E Z H 2^{f l / f 1}$ mice, the PDX1-positive cells were negative for $\mathrm{p} 16^{\mathrm{INK} 4 \mathrm{~A}}$ expression (Fig. 4B). In contrast, a pronounced up-regulation of $\mathrm{p} 16^{\mathrm{INK} 4 \mathrm{~A}}$ was detected in PDX1-positive metaplastic

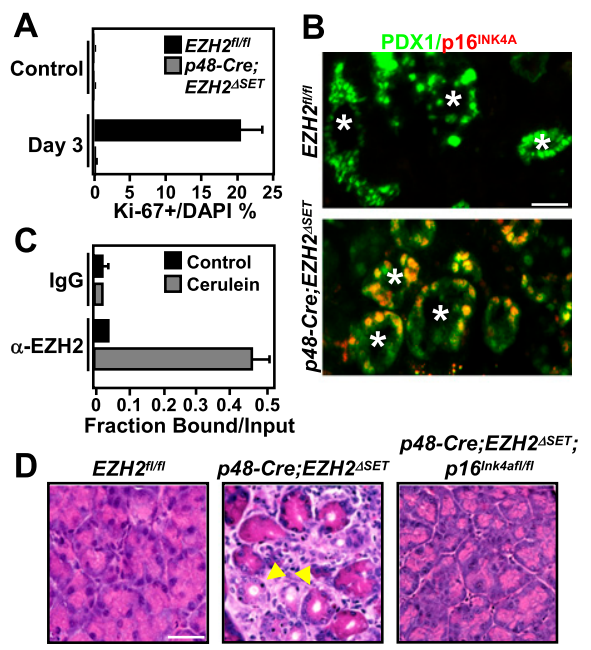

Figure 4. EZH2 is required for the proliferative expansion step during pancreatic regeneration. (A) Quantification of Ki67-positive cells at day 3 after final cerulein injection shows a proliferation defect in p48-Cre;EZH2 $2^{\Delta S E T}$ pancreata. Results represent the means $\pm S D$ of three independent determinations in two mice per genotype. $(B)$ Double immunofluorescence analysis of $\mathrm{p} 16^{\mathrm{INK} 4 \mathrm{~A}}$ and PDX1 demonstrates that $16^{\mathrm{INK} 4 \mathrm{~A}}$ is suppressed in the PDX1positive metaplastic epithelium (marked by asterisks) in $E Z H 2^{f 1 / f 1}$ but not p48-Cre;EZH2 $2^{\triangle S E T}$ pancreata. Images shown are representative of data obtained from three mice per genotype. Bar, $50 \mu \mathrm{m}$. (C) Chromatin immunoprecipitation analysis of the $p 16^{\text {Ink }} 4 a$ locus in $E Z H 2^{f l / f 1}$ pancreata harvested at day 3 after the final cerulein injection demonstrates recruitment of EZH2 to the $p 16^{\text {Ink } 4 a}$ promoter during regeneration. Results represent the means $\pm S D$ of three independent determinations in two mice per genotype. (D) Pancreata from EZH2 $2^{f 1 / f 1}$, p48-Cre;EZH2 $2^{\Delta S E T}$, and $p 48$ Cre;EZH2 $2^{\Delta S E T} ; p 16^{\text {Ink } 4 a f 1 / f 1}$ mice were harvested on day 9 following the final cerulein injection. H\&E-stained sections show that impaired regeneration is rescued in p48-Cre;EZH2 ${ }^{\Delta S E T} ; p 16^{\text {Ink } 4 a f 1 / f 1}$ pancreata. Selected metaplastic lesions are indicated by arrowheads. Images shown are representative of data obtained from three animals per genotype. Bar, $50 \mu \mathrm{m}$. 
cells from $p 48-C r e$; $E Z H 2^{\Delta S E T}$ pancreata (Fig. 4B), suggesting that EZH2 is required to maintain transcriptional silencing of $\mathrm{p} 16^{\mathrm{INK} 4 \mathrm{~A}}$ expression in the PDX1-positive metaplastic epithelium of the regenerating pancreas. To determine whether this effect is mediated by the recruitment of EZH2 to the $p 16^{\text {Ink4a }}$ locus, we performed chromatin immunoprecipitation assays. A pronounced enrichment of EZH2 at the $p 16^{\text {Ink } 4 a}$ promoter was observed in cerulein-treated $E Z H 2^{f l / f 1}$ but not in $p 48$ Cre;EZH2 ${ }^{\triangle S E T}$ pancreata (Fig. 4C; Supplemental Fig. 8A). Thus, the contribution of EZH2 to tissue renewal in the setting of pancreatic regeneration might be mediated through preventing aberrant expression of $\mathrm{p} 16^{\mathrm{INK} 4 \mathrm{~A}}$. To test this idea directly, p48-Cre;EZH2 ${ }^{\Delta S E T}$ mice were crossed to $p 16^{\text {Ink } 4 a f 1 / f 1}$ mice to generate mice of the genotype $p 48$-Cre;EZH2 ${ }^{\Delta S E T} ;$ p16 $6^{\text {Ink } 4 a f 1 / f 1}$. As shown (Fig. 4D; Supplemental Fig. 8B), the defective regenerative response to cerulein administration was rescued in pancreata of $p 48$-Cre;EZH2 $2^{\Delta S E} ; p 16^{\text {Ink } 4 \text { afl/f1 }}$ animals. These results indicate that $\mathrm{EZH} 2$ is required during pancreatic repair to initiate proliferation of the PDX1-positive metaplastic compartment by suppressing $\mathrm{p} 16^{\mathrm{INK} 4 \mathrm{~A}}$. We conclude that loss of EZH2 permits sustained p16 ${ }^{\mathrm{INK} 4 \mathrm{~A}}$ expression in the metaplastic progenitor cells, blocking their proliferation and redifferentiation into acini, thereby bringing regeneration to a halt.

Perturbations in pathways that control tissue regeneration and renewal have been linked to increased risk for neoplastic conversion (Coussens and Werb 2002). In light of the observed role of EZH2 in pancreatic regeneration, we set out to investigate the consequence of EZH2 loss in the context of KRas ${ }^{\mathrm{G} 12 \mathrm{D}}$-induced pancreatic neoplasia. To this end, $L S L-K_{r a s}{ }^{G 12 D}$ mice were crossed to $p 48$-Cre;EZH2 $2^{\Delta S E T}$ mice to generate mice of the genotype p48-Cre;Kras ${ }^{G 12 D} ; E Z H 2^{4 S E T}$. These mice were born at the expected Mendelian ratios but displayed a dramatic reduction in survival, reaching terminal morbidity between the ages of 12 and 16 wk $(n=12)$. No animals of other genotypes showed any signs of distress at this age. Gross inspection of dissected $p 48$ Cre;Kras ${ }^{G 12 D} ; E Z H 2^{\Delta S E T}$ pancreata revealed dramatic alterations in the overall appearance of the organ. At 1 mo of age, a somewhat enlarged, nodular, and edematous
pancreas was seen in the p48-Cre;Kras ${ }^{G 12 D} ; E Z H 2^{\Delta S E T}$ mice (Supplemental Fig. 9). In comparison, the pancreas from $p 48$-Cre; Kras ${ }^{G 12 D}$ of the same age appeared normal. Over the next $2 \mathrm{mo}$, p48-Cre;Kras ${ }^{G 12 D}$;EZH2 $2^{\Delta S E T}$ pancreata underwent progressive atrophy, whereas pancreata from $p 48$-Cre;Kras ${ }^{G 12 D}$ increased in size and displayed macroscopic nodularity (Supplemental Fig. 9). Histological analysis of pancreatic tissue from 1- and 2-mo-old p48Cre;Kras ${ }^{G 12 D} ; E Z H 2^{\Delta S E T}$ mice demonstrated a significant impact of EZH2 deficiency on neoplastic progression. Whereas $p 48$-Cre; Kras ${ }^{G 12 D}$ pancreata developed predominantly focal low-grade PanINs in the course of $2 \mathrm{mo}, \mathrm{p} 48$ Cre;Kras ${ }^{G 12 D}$;EZH2 $2^{\Delta S E T}$ pancreata displayed not only a marked enhancement in the number of early mPanINs, but also advanced PanIN lesions as early as 1 mo of age (Fig. 5A; Supplemental Fig. 10A,B). Severe atrophy of $p 48$ Cre;Kras ${ }^{G 12 D} ; E Z H 2^{\Delta S E T}$ pancreata precluded comparative analysis beyond the age of 3 mo. However, as a reference, $p 48$-Cre;Kras ${ }^{G 12 D}$ mice develop advanced mPanINs with a mean latency of 6 mo (Aguirre et al. 2003). These observations demonstrate that loss of EZH2 accelerates initiation and neoplastic progression of KRas ${ }^{\mathrm{G} 12 \mathrm{D}}$-driven PanINs.

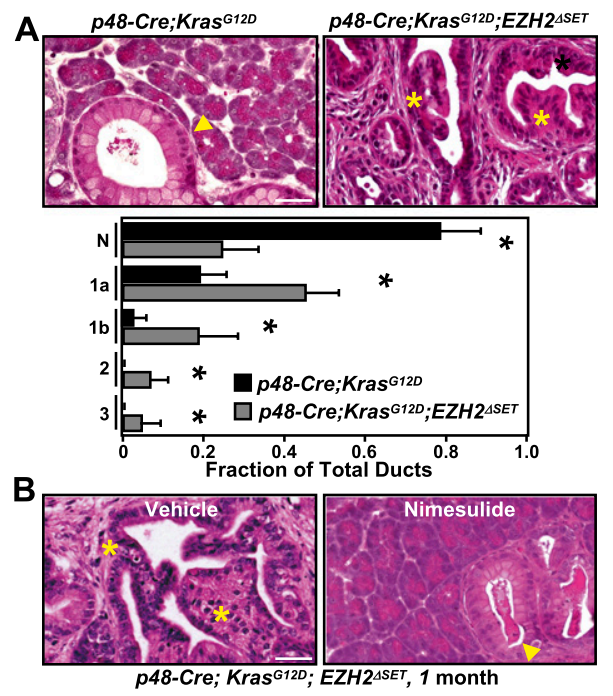

Figure 5. EZH2 deficiency accelerates PanIN progression. $(A$, top panel) H\&E-stained pancreatic sections from 1-mo-old $p 48$ Cre; Kras ${ }^{G 12 D}$ and p48-Cre; Kras ${ }^{G 12 D} ; E Z H 2^{\Delta S E T}$ mice show accelerated PanIN progression upon EZH2 loss. (Arrowhead) Early PanIN lesions; $\left({ }^{*}\right)$ advanced PanIN lesions. Bar, $50 \mu \mathrm{m}$. (Bottom panel) Quantification of histological progression of PanINs in $p 48$ Cre; Kras ${ }^{G 12 D}$ and p48-Cre; Kras ${ }^{G 12 D} ; E Z H 2^{4 S E T}$ mice. (N) Normal; (1, 1a, 1b, 2, 3) PanIN stage. Results represent the means \pm SD of lesions per section identified (five mice per genotype). $\left(^{\star}\right) P<0.05$. (B) H\&E analysis shows that administration of nimesulide abrogates accelerated PanIN progression in p48-Cre; Kras ${ }^{G 12 D} ; E Z H 2^{\Delta S E T}$ mice. (Arrowhead) Early PanIN lesions; $\left(^{\star}\right)$ advanced PanIN lesions. Bar, $50 \mu \mathrm{m}$.

Pancreatic injury in the setting of oncogenic KRas expression has been shown to hasten PanIN development and progression (Guerra et al. 2007). This promoting effect has been attributed to the inflammatory response that ensues as a consequence of tissue damage (Guerra et al. 2007). Since loss of EZH2 is associated with impaired regeneration and, by default, prolonged injury, we reasoned that acceleration of neoplastic progression in p48-Cre;Kras ${ }^{G 12 D}$;EZH2 ${ }^{\Delta S E T}$ mice could be due to an inflammatory microenvironment that is established in the pancreata of these animals. To test this idea, we first sought to characterize the stromal changes that accompany PanIN progression in p48-Cre;Kras ${ }^{G 12 D} ; E Z H 2^{\Delta S E T}$ mice. A marked increase in $\mathrm{CD} 45^{+}$cells was observed in pancreata of 1 -mo-old p48-Cre;Kras G12D $; E Z H 2^{\Delta S E T}$ mice in comparison with p48-Cre; Kras ${ }^{G 12 D}$ mice (Supplemental Fig. 11A). This increase reflected predominantly the accumulation of $\mathrm{CD}_{1} 1 \mathrm{~b}^{+}$macrophages, $\mathrm{Gr}-1^{+}$neutrophils, and $\mathrm{CD} 11 \mathrm{c}^{+}$dendritic cells, suggesting preferential recruitment of components of the innate immune system (Supplemental Fig. 11B). Consistent with the overrepresentation of inflammatory immunocytes, pancreata from p48-Cre;Kras ${ }^{G 12 D} ; E Z H 2^{4 S E T}$ mice displayed widespread collagen deposition and desmoplasia, as indicated by activation of smooth muscle actin (Supplemental Fig. 11C). The progressive nature of the fibrotic reaction likely contributes to the severe atrophy of the pancreas observed as the p48-Cre;Kras ${ }^{G 12 D} ; E Z H 2^{4 S E T}$ mice age.

To further examine whether the heightened inflammatory conditions that arise as a result of EZH2 loss were critical for advancing PanIN progression, we tested the effect of the anti-inflammatory Cox- 2 inhibitor nimesulide on the 
histological presentation of $p 48-C r e ; \operatorname{Kras}^{G 12 D} ; E Z H 2^{\Delta S E T}$ pancreata. One month of treatment with a nimesulidecontaining diet completely ameliorated the inflammatory response and restricted PanIN progression to the level seen in p48-Cre; Kras ${ }^{G 12 D}$ mice (Fig. 5B; Supplemental Fig. 12A). Significantly, during this interval, evolving PanIN lesions in p48-Cre;Kras ${ }^{\text {12D }}$ mice were not affected by nimesulide treatment (Supplemental Fig. $12 \mathrm{~A}, \mathrm{~B})$, suggesting that the inhibitor exerts its effects predominantly by interfering with stromal activation, rather than through a cell-autonomous mechanism. Together, these observations implicate EZH2 in constraining oncogenic KRas-driven neoplastic progression through homeostatic mechanisms that control pancreatic regeneration and prevent unscheduled inflammation. By analogy to the injury repair response triggered by cerulein, our findings suggest that the process of PanIN development could be accompanied by cycles of tissue injury and regeneration. The molecular pathways that mediate the disruptive effects of neoplastic lesions on pancreatic tissue integrity remain to be established.

The results presented in this study provide evidence in support of a direct role for EZH2-mediated suppression of p $16^{\mathrm{INK} 4 \mathrm{~A}}$ expression in the regeneration of the exocrine pancreas. The epigenetic modification of $p 16^{\text {Ink } 4 a}$ by EZH2 is specifically required to control the proliferative potential of PDX1-positive progenitor cells, which accumulate transiently in metaplastic lesions that are formed following pancreatic injury. A similar lineage-specific regulation of cell proliferation by EZH2 has been recently described in the context of epidermal progenitors (Ezhkova et al. 2011). Although in the absence of comprehensive transcriptional profiling analysis we cannot unequivocally rule out the involvement of additional EZH2 targets in the regenerative process, the observation that p $16^{\mathrm{INK} 4 \mathrm{~A}}$ loss completely rescues the defect in regeneration displayed by EZH2-deleted animals argues for a critical involvement of the EZH $2 / \mathrm{p} 6^{\mathrm{INK} 4 \mathrm{~A}}$ axis in this process.

Based on in vitro studies, EZH2 has been predicted to play an oncogenic role as it promotes proliferation of prostate (Karanikolas et al. 2009), breast (Collett et al. 2006), and pancreatic (Ougolkov et al. 2008) cancer cells in culture. Our present findings suggest that in vivo, EZH2 might play an opposing role; namely, restraining the oncogenic process in the exocrine pancreas. Given the abundance of experimental and clinical evidence linking chronic pancreatic injury and neoplasia, we propose that EZH2 counteracts the proneoplastic effects of pancreatic injury by promoting regenerative proliferation. Further studies will be required to determine whether EZH2 affects the neoplastic process through additional cell-intrinsic mechanisms that are not directly related to tissue regeneration as well as whether it plays a similar role in other organs.

\section{Materials and methods}

See the Supplemental Material for more information.

\section{Mice and treatments}

Pancreatic deletion of EZH2 was obtained by crossing p48-Cre mice (Kawaguchi et al. 2002) to EZH2 $2^{f l / f 1}$ mice (Su et al. 2003).p48-Cre;Kras ${ }^{G 12 D}$ mice were generated by crossing p48-Cre mice to LSL-Kras ${ }^{G 12 D}$ mice

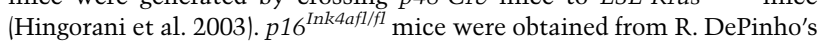
laboratory (Monahan et al. 2007). EZH2 was deleted in the Kras ${ }^{G 12 D}$ background by crossing p48-Cre;EZH2 $2^{f l / f l}$ mice to $L S L-\operatorname{Kras}^{G 12 D} ; E Z H 2^{f l / f l}$ mice. For induction of pancreatitis, cerulein $(50 \mu \mathrm{g} / \mathrm{kg})$ was administered by i.p. injections hourly, seven times a day, $3 \mathrm{~d}$ a week for three consecutive weeks. Pancreata were harvested at the indicated time points. Control refers to saline injections using the same schedule as cerulein and harvested at day 1 after final injection. Where indicated, a nimesulideenriched (Cayman Chemicals) diet $(0.4 \mathrm{~g} / \mathrm{kg}$ diet; Dyets) was fed to parents of mice upon birth to start treatment of pups as early as possible. All animal care and procedures followed National Institutes of Health guidelines and were approved by the Institutional Animal Care and Use Committee at New York University School of Medicine.

\section{Western blotting}

Proteins were fractionated by electrophoresis on sodium dodecyl sulfate (SDS) polyacrylamide gels followed by transfer onto nitrocellulose membranes. Membranes were probed using standard Western blotting protocols followed by visualization using the Odyssey infrared imaging system (Li-Cor). The primary antibodies used were rabbit anti-EZH1 (Margueron et al. 2008), rabbit anti-EZH2 (Kuzmichev et al. 2004), rabbit anti-SUZ12 (Kuzmichev et al. 2004), mouse anti-ERK2 (Upstate Biotechnology), mouse anti-vinculin (Sigma), goat anti-amylase (Santa Cruz Biotechnology), and rat anti-CK19 (TROMA-III-c, developed by R. Kemler, obtained from the Developmental Studies Hybridoma Bank under the auspices of the National Institute of Child Health and Human Development [NICHD] and maintained by The University of Iowa, Department of Biology, Iowa City).

\section{Acknowledgments}

We are grateful to A. Tarakhovsky (The Rockefeller University), T. Jacks (Massachusetts Institute of Technology), R.A. DePinho (The University of Texas, MD Anderson Cancer Center), and C.V. Wright (Vanderbilt University) for mice. We also thank Jun Liang Zhou and Deborah Hernandez for their technical assistance, C.H. Hajdu and the Tissue Acquisition and Biorepository Service (TABS) for help with human samples, the Histopathology Core for immunohistochemical staining, and members of the Bar-Sagi laboratory for their comments on the manuscript. TABS and the Histopathology Core are supported in part by 5P30CA016087-33 from the National Cancer Institute. This work was supported by grants to D.B.-S. (NIH CA055360 and AACR 08-60-25BARS), D.R. (Howard Hughes Medical Institute and NIH GM64844 and GM37120), J.M.-S.C. (NIH 1F30AA017344), R.M. (Department of Defense PC050535), G.M. (NIH DK085278 and CA155649), and Y.P.-G. (Irvington Institute Fellowship Program of the Cancer Research Institute).

\section{References}

Aguirre AJ, Bardeesy N, Sinha M, Lopez L, Tuveson DA, Horner J, Redston MS, DePinho RA. 2003. Activated Kras and Ink4a/Arf deficiency cooperate to produce metastatic pancreatic ductal adenocarcinoma. Genes Dev 17: 3112-3126.

Chen H, Gu X, Su IH, Bottino R, Contreras JL, Tarakhovsky A, Kim SK. 2009. Polycomb protein Ezh2 regulates pancreatic $\beta$-cell Ink4a/Arf expression and regeneration in diabetes mellitus. Genes Dev 23: 975 985.

Collett K, Eide GE, Arnes J, Stefansson IM, Eide J, Braaten A, Aas T, Otte AP, Akslen LA. 2006. Expression of enhancer of zeste homologue 2 is significantly associated with increased tumor cell proliferation and is a marker of aggressive breast cancer. Clin Cancer Res 12: 1168-1174.

Coussens LM, Werb Z. 2002. Inflammation and cancer. Nature 420: 860867.

Desai BM, Oliver-Krasinski J, De Leon DD, Farzad C, Hong N, Leach SD, Stoffers DA. 2007. Preexisting pancreatic acinar cells contribute to acinar cell, but not islet $\beta$ cell, regeneration. J Clin Invest 117: 971-977.

Ezhkova E, Pasolli HA, Parker JS, Stokes N, Su IH, Hannon G, Tarakhovsky A, Fuchs E. 2009. Ezh2 orchestrates gene expression for the stepwise differentiation of tissue-specific stem cells. Cell 136: 1122-1135.

Ezhkova E, Lien WH, Stokes N, Pasolli HA, Silva JM, Fuchs E. 2011. EZH1 and EZH2 cogovern histone $\mathrm{H} 3 \mathrm{~K} 27$ trimethylation and are essential for hair follicle homeostasis and wound repair. Genes Dev 25: 485-498. 


\section{Mallen-St. Clair et al.}

Fendrich V, Esni F, Garay MV, Feldmann G, Habbe N, Jensen JN, Dor Y, Stoffers D, Jensen J, Leach SD, et al. 2008. Hedgehog signaling is required for effective regeneration of exocrine pancreas. Gastroenterology 135: 621-631.

Guerra C, Schuhmacher AJ, Canamero M, Grippo PJ, Verdaguer L, PerezGallego L, Dubus P, Sandgren EP, Barbacid M. 2007. Chronic pancreatitis is essential for induction of pancreatic ductal adenocarcinoma by K-Ras oncogenes in adult mice. Cancer Cell 11: 291-302.

Hingorani SR, Petricoin EF, Maitra A, Rajapakse V, King C, Jacobetz MA Ross S, Conrads TP, Veenstra TD, Hitt BA, et al. 2003. Preinvasive and invasive ductal pancreatic cancer and its early detection in the mouse. Cancer Cell 4: 437-450.

Jensen JN, Cameron E, Garay MV, Starkey TW, Gianani R, Jensen J. 2005 Recapitulation of elements of embryonic development in adult mouse pancreatic regeneration. Gastroenterology 128: 728-741.

Karanikolas BD, Figueiredo ML, Wu L. 2009. Polycomb group protein enhancer of zeste 2 is an oncogene that promotes the neoplastic transformation of a benign prostatic epithelial cell line. Mol Cancer Res 7: 1456-1465.

Kawaguchi Y, Cooper B, Gannon M, Ray M, MacDonald RJ, Wright CV. 2002. The role of the transcriptional regulator Ptfla in converting intestinal to pancreatic progenitors. Nat Genet 32: 128-134.

Koizumi M, Doi R, Toyoda E, Masui T, Tulachan SS, Kawaguchi $\mathrm{Y}$ Fujimoto K, Gittes GK, Imamura M. 2003. Increased PDX-1 expression is associated with outcome in patients with pancreatic cancer Surgery 134: 260-266.

Kuzmichev A, Jenuwein T, Tempst P, Reinberg D. 2004. Different EZH2 containing complexes target methylation of histone $\mathrm{H} 1$ or nucleosomal histone H3. Mol Cell 14: 183-193.

Margueron R, Reinberg D. 2011. The Polycomb complex PRC2 and its mark in life. Nature 469: 343-349.

Margueron R, Li G, Sarma K, Blais A, Zavadil J, Woodcock CL, Dynlacht BD, Reinberg D. 2008. Ezh1 and Ezh2 maintain repressive chromatin through different mechanisms. Mol Cell 32: 503-518.

Monahan KB, Rozenberg GI, Krishnamurthy J, Johnson SM, Liu W, Bradford MK, Horner J, Depinho RA, Sharpless NE. 2007. Somatic p16(INK4a) loss accelerates melanomagenesis. Oncogene 29: 58095817.

Ougolkov AV, Bilim VN, Billadeau DD. 2008. Regulation of pancreatic tumor cell proliferation and chemoresistance by the histone methyltransferase enhancer of zeste homologue 2. Clin Cancer Res 14: 67906796.

Sawey ET, Johnson JA, Crawford HC. 2007. Matrix metalloproteinase 7 controls pancreatic acinar cell transdifferentiation by activating the Notch signaling pathway. Proc Natl Acad Sci 104: 19327-19332.

Siveke JT, Lubeseder-Martellato C, Lee M, Mazur PK, Nakhai H, Radtke F, Schmid RM. 2008. Notch signaling is required for exocrine regeneration after acute pancreatitis. Gastroenterology 134: 544-555.

Su GH, Hruban RH, Bansal RK, Bova GS, Tang DI, Shekher MC, Westerman AM, Entius MM, Goggins M, Yeo CJ, et al. 1999. Germline and somatic mutations of the STK11/LKB1 Peutz-Jeghers gene in pancreatic and biliary cancers. Am J Pathol 154: 1835-1840.

Su IH, Basavaraj A, Krutchinsky AN, Hobert O, Ullrich A, Chait BT Tarakhovsky A. 2003. Ezh2 controls B cell development through histone H3 methylation and Igh rearrangement. Nat Immunol 4: 124131.

Thayer SP, di Magliano MP, Heiser PW, Nielsen CM, Roberts DI, Lauwers GY, Qi YP, Gysin S, Fernandez-del Castillo C, Yajnik V, et al. 2003. Hedgehog is an early and late mediator of pancreatic cancer tumorigenesis. Nature 425: 851-856.

Whitcomb DC. 2004. Inflammation and cancer V. Chronic pancreatitis and pancreatic cancer. Am I Physiol Gastrointest Liver Physiol 287: G315-G319. doi: 10.1152/ajpgi.00115.2004. 


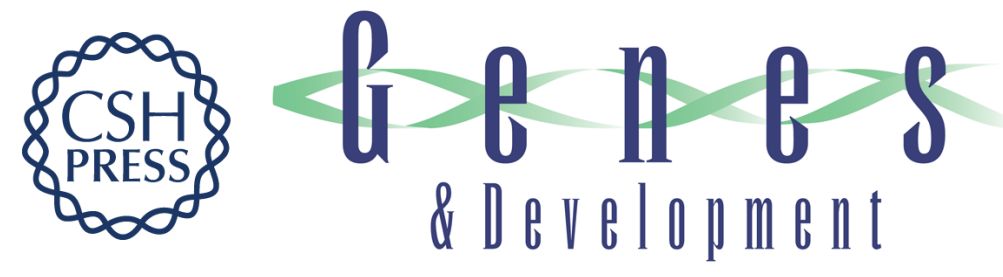

\section{EZH2 couples pancreatic regeneration to neoplastic progression}

Jon Mallen-St. Clair, Rengin Soydaner-Azeloglu, Kyoung Eun Lee, et al.

Genes Dev. 2012, 26:

Access the most recent version at doi:10.1101/gad.181800.111

Supplemental
Material $\quad$ ttp://genesdev.cshlp.org/content/suppl/2012/03/05/26.5.439.DC1

References This article cites 25 articles, 7 of which can be accessed free at: http://genesdev.cshlp.org/content/26/5/439.full.html\#ref-list-1

License

Email Alerting Receive free email alerts when new articles cite this article - sign up in the box at the top Service right corner of the article or click here.

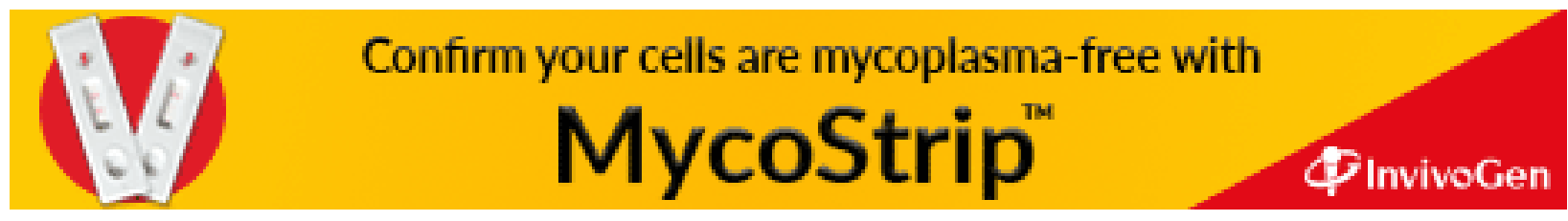

